\title{
TUNNEL ILLUMINATION: ALGORITHM TO DETERMINE STANDARD VALUES OF LUMINANCE IN TUNNEL ZONES USING C PROGRAMMING LANGUAGE APPROACH
}

\author{
Canan Perdahci ${ }^{1}$, Onur Hasanoglu ${ }^{2}$, and YahyaSogodok ${ }^{3}$ \\ Kocaeli University, Department of Electrical Engineering, Kocaeli, Turkey \\ Emails: ${ }^{1}$ perdahci@kocaeli.edu.tr, ${ }^{2}$ onurhasanoglu97@gmail.com, \\ 3yahya.s7495@gmail.com
}

\begin{abstract}
Tunnel illumination design involves complex mathematical computation, cumbersome, and tedious to perform when done by hand. This paper proposes an algorithm to compute luminance and luminous flux of the different zones of the tunnel by following the International Commission on Illumination (CIE88:2004) standard design guidelines using the $C$ programming language. The luminance and the luminous flux values of all the zones were calculated and compared with the CIE standard. The energy consumption of each zone is equally found to control and reduce energy wastage and payment of unwanted bills. The threshold zone length $L_{t h}$, average luminance in the first half $L_{a v}$, and the last half of the threshold zone are $40 \mathrm{~m}, 400 \mathrm{~cd} / \mathrm{m}^{2}$, and 240 $\mathrm{cd} / \mathrm{m}^{2}$, respectively, while the average luminance of the transition and interior zones are, respectively, $95.87 \mathrm{~cd} / \mathrm{m}^{2}$ and $5.86 \mathrm{~cd} / \mathrm{m}^{2}$. The results obtained were satisfying. The computational results showed that the proposed Algorithm could be used to design an efficient tunnel illumination system with less energy waste.
\end{abstract}

Keywords: energy-saving, tunnel illumination, luminance, $C$ programming, LED

\section{INTRODUCTION}

In tunnel design, the crucial part is the illumination of the tunnel. The improper illumination of the tunnel leads to essential losses in lives and proper- ties. Therefore, the International Commission on Illumination (CIE88:2004) [1] standard and the European Committee for Standardization (CEN) proposed some guidelines, such as the speed limit of the tunnel, surrounding luminance, length of the tunnel, and so on. To consider during tunnel illumination system design to ensure the security and safety of the driver when entering and exiting the tunnel. Since then, many researchers have proposed different optimization design methods to satisfy these guidelines. The optimization method should consider the technical design of the illumination system, such as the luminaries' parameters and spacing between them, the luminous flux and luminance inside and outside the tunnel, installation and maintenance cost [2]. For example, the study done in [3] proposed an optimization approach to tackle the issue related to the light distribution of luminaires design. The luminaires parameters were expressed as a linear optimization function, considering the luminous intensity as the objective function needed to construct the luminance curve. The result showed that the optimization algorithm was efficient and could improve the luminaries' photometric parameters. In similar research, the parameters optimization model (POM) was studied for the interior zone of the tunnel by considering the optimization variables of the luminaries, such as installation height, longitudinal spacing, power, and elevation angle. The obtained outcome was compared with a similar work of the Yanlieshan tunnel illumi- 
Table 1. The Average Luminance Values of the Access Zone Are Perceived at $20^{\circ}$

\begin{tabular}{|c|c|c|c|c|c|c|c|c|c|c|c|c|c|c|c|c|}
\hline \multirow{5}{*}{\begin{tabular}{c|} 
\\
THE VIEW \\
LUMI- \\
NANCE
\end{tabular}} & \multicolumn{16}{|c|}{$20^{\circ}$ CONICAL SKY VIEW } \\
\hline & \multicolumn{4}{|c|}{$35 \%$} & \multicolumn{4}{|c|}{$25 \%$} & \multicolumn{4}{|c|}{$10 \%$} & \multicolumn{4}{|c|}{$0 \%$} \\
\hline & \multicolumn{2}{|c|}{ NORMAL } & \multicolumn{2}{|c|}{ SNOW } & \multicolumn{2}{|c|}{ NORMAL } & \multicolumn{2}{|c|}{ SNOW } & \multicolumn{2}{|c|}{ NORMAL } & \multicolumn{2}{|c|}{ SNOW } & \multicolumn{2}{|c|}{ NORMAL } & \multicolumn{2}{|c|}{ SNOW } \\
\hline & few & much & few & much & few & much & few & much & few & much & few & much & few & much & few & much \\
\hline & $(* 1)$ & $(* 1)$ & $(* 1)$ & $(* 1)$ & $(* 1)$ & $(* 1)$ & $(* 1)$ & $(* 1)$ & $(* 2)$ & $(* 2)$ & $(* 3)$ & $(* 3)$ & $(* 2)$ & $(* 2)$ & $(* 3)$ & $(* 3)$ \\
\hline $\mathrm{SD} 60 \mathrm{~m}$ & $(* 4)$ & $(* 4)$ & $(* 4)$ & $(* 4)$ & 4000 & 5000 & 4000 & 5000 & 2500 & 3500 & 3000 & 3500 & 1500 & 3000 & 1500 & 4000 \\
\hline $\begin{array}{l}\text { STOPPING } \\
\text { DISTANCE } \\
(100-160) \mathrm{m}\end{array}$ & 4000 & 6000 & 4000 & 6000 & 4000 & 6000 & 4000 & 6000 & 3000 & 4500 & 3000 & 5000 & 2500 & 5000 & 2500 & 5000 \\
\hline
\end{tabular}

nation system. The POM method showed a considerable energy-saving than the latter [4].

Moreover, apart from the proposed optimization, some researchers proposed some technical and economical methods to design tunnels effectively with less cost and energy efficient. As an example, [5] instead focused on the material used to construct the pavement of the tunnel, which has a remarkable impact on the installation and maintenance cost. For the first year, the study shows that concrete pavement was much more economical than the flexible structure for a tunnel of length equal to or shorter than $1000 \mathrm{~m}$. In contrast, for a $1200 \mathrm{~m}$ and above the tunnel, the asphalt pavement offered a better financial and technical result than its counterpart concrete pavement. Furthermore, a genetic algorithm was used in [6] for automating tunnel illumination system design to ensure the security and safety of the driver through clear visual perception in the tunnel. The luminance of the different tunnel zones was obtained using the developed software, which systematically selects the suitable light based on the design specifications provided by the user. Another optimal solution was proposed in [7], whereby tunnel optimization was done using a computer software. The optimal solution was tested on an interior zone of $10 \mathrm{~km}$ to calculate the luminance, and it showed that, considerable amount of energy was saved. All the above-mentioned optimization problems were applicable to one or two tunnel zones only, so this study proposed an algorithm to calculate the luminance of all the tunnel zones. The $C$ programming language was used to implement the proposed Algorithm to obtain the luminance and luminous flux of different tunnel zones. The algorithm was assessed on 2 different tunnels to verify for its efficiency.

In the above literature, there are various professional programs, such as Litestar 4D, ReluxTun- nel, Agi32, etc. for tunnel illumination calculation. Still, few studies allow a new illumination designer to understand the algorithm in the backend. Understanding the algorithm can lead to better improvement of the algorithm by the illumination designer, especially for new tunnel lighting designers. The algorithm we have developed is entirely based on tunnel illumination theoretical knowledge and standard using the $C$ programming language. $C$ programming language is among the most widely used programming languages for apps development and program software, and easy to install, understandable and offers conveniences to its users. Therefore, the $C$ programming language was used to promote our algorithm, which is developed to be run in all $C$ programming IDE environments and supply amenities to its users.

\section{MATERIAL AND METHOD}

\subsection{Luminaires}

There are many luminaires used in tunnel illumination. For many years, the most used luminaires were ones with the high-pressure sodium lamp (HPS) and later with the compact fluorescent lamps (CFL). However, these lamps are not energy efficient, and hence lots of energy is being consumed. Therefore, the lamps are not economical. The need for more efficient and energy-efficient illumination led to light emitting diode (LED) luminaires. This luminaire has excellent photometric properties as well as consumes less energy. For this work, the calculation was made considering the LED luminaires for calculating the CIE standard values. The choice of these luminaires is due to their high-quality features and minimal energy consumption, unlike other luminaires. For the Alanya tunnel, HPS was considered and supplied a counter beam for the illumina- 
Table 2. Tunnel Data

\begin{tabular}{|c|c|c|c|}
\hline & CASE1 & CASE2 & CASE3 \\
\hline & CIE standard & Alanya tunnel & Trabzon tunnel \\
\hline Length of the tunnel $(l)$ & $5000 \mathrm{~m}$ & $4060 \mathrm{~m}$ & $1702 \mathrm{~m}$ \\
\hline Access zone luminance $\left(L_{20}\right)$ & $4000 \mathrm{~cd} / \mathrm{m}^{2}$ & $4800 \mathrm{~cd} / \mathrm{m}^{2}$ & $5000 \mathrm{~cd} / \mathrm{m}^{2}$ \\
\hline Luminaire type & LED & $\mathrm{HPS}$ & $\mathrm{HPS}$ \\
\hline Luminaire luminous flux & $28000 \mathrm{~lm}$ & $34000 \mathrm{~lm}$ & $28000 \mathrm{~lm}$ \\
\hline Luminaire distribution curve type & Symmetric & Counter beam & Counter beam \\
\hline Luminaire surface area & $0.08 \mathrm{~m}^{2}$ & $0.1845 \mathrm{~m}^{2}$ & $0.08 \mathrm{~m}^{2}$ \\
\hline Luminaire power & $200 \mathrm{~W}$ & $150 \mathrm{~W}$ & $200 \mathrm{~W}$ \\
\hline The speed limit of the tunnel & $80 \mathrm{~km} / \mathrm{h}$ & $90 \mathrm{~km} / \mathrm{h}$ & $80 \mathrm{~km} / \mathrm{h}$ \\
\hline
\end{tabular}

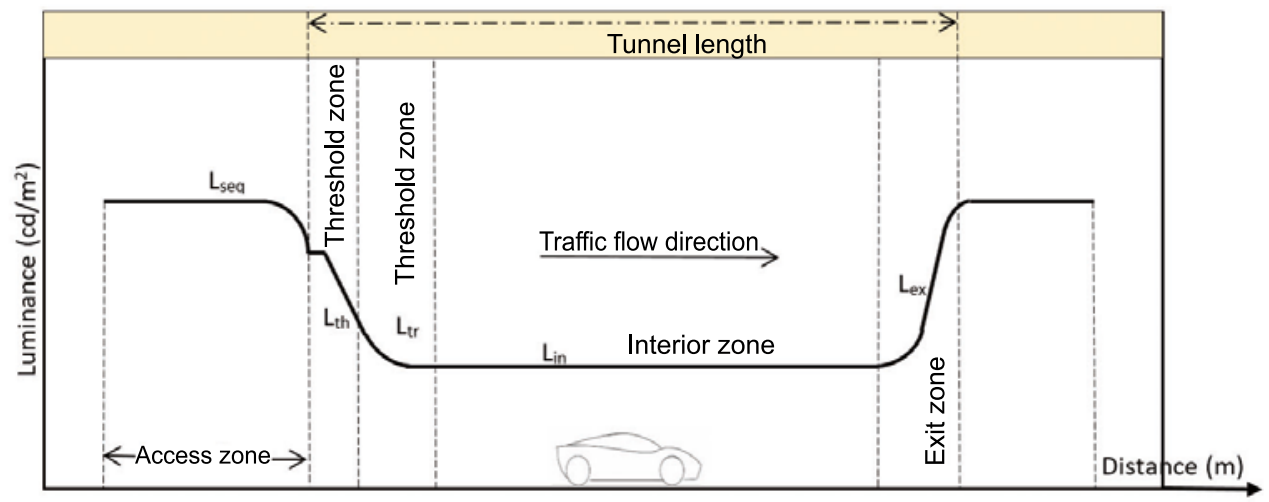

Fig. 1. Zones in a tunnel [8] tion system in the tunnel. The features of the luminaires are described in Table 2.

\subsection{Zones in the Tunnel}

The tunnel is divided into access, threshold, transition, interior, and exit zones. Fig. 1 below shows the zones for a one-way tunnel.

\subsection{Luminance Distribution along the Tunnel}

The luminance varies throughout the tunnel, with the highest value around the tunnel's threshold and exit zones. These high luminance values are due to the 'black-hole' and 'white-hole' phenomenon encounter by drivers when entering and exiting the tunnel. Thus, these two zones consume enormous energy. Fig. 2 shows the distribution of the luminance in the threshold and transition zones of the tunnel.

The luminance of the access zone $L_{20}$ is the luminance inside the conical area focused on a point one-fourth of the tunnel entrance's height and con- necting the boundaries of a $20^{\circ}$ from the driver's eye entering the tunnel $[3,6]$. Thus, the $L_{20}$ is an essential factor for finding the luminance of the threshold zone. In Table 1, it is the average luminance values of the access zone are perceived at $20^{\circ}$.

The average road surface luminance $\left(L_{t h}\right)$ of the threshold zone, as shown in Fig. 1, represents the threshold zone luminance [9].

$$
L_{t h}=k L_{20} \text {. }
$$

The ratio between the average road surface luminance $\left(L_{t h}\right)$ in the first section of the threshold zone and the luminance $\left(L_{20}\right)$ was measured at a distance equal to the stopping distance in front of the tunnel in the access zone.

Illumination systems that meet the $0.2<L /$ House $<0.6$ conditions are rarely met. Even with such illumination, the $k$ values recommended for the symmetrical design are accepted.

The road surface luminance's average value anywhere in the transition zone is called the transition zone luminance $\left(L_{t r}\right)$ [11]. The formula for the computation of the $L_{t r}$ is given in eq. (1). 
Table 3. The Tunnel Detailed Results Using CIE Standard Values

\begin{tabular}{|c|c|c|c|c|}
\hline Tunnel zone detail & \multicolumn{2}{|c|}{ Threshold } & Transition & Interior \\
\hline Section & First & Second & & \\
\hline Length & $20 \mathrm{~m}$ & $20 \mathrm{~m}$ & & \\
\hline Average luminance & $240 \mathrm{~cd} / \mathrm{m}^{2}$ & $144 \mathrm{~cd} / \mathrm{m}^{2}$ & $95.87 \mathrm{~cd} / \mathrm{m}^{2}$ & $5.86 \mathrm{~cd} / \mathrm{m}^{2}$ \\
\hline Luminous flux intensity coefficient & 0.22 & 0.20 & 0.09 & 0.01 \\
\hline Energy consumption & $6.45 \%$ & $6.02 \%$ & $51.57 \%$ & $35.95 \%$ \\
\hline
\end{tabular}

Table 4. Calculation Results for Alanya Tunnel

\begin{tabular}{|c|c|c|c|c|}
\hline Tunnel zone detail & \multicolumn{2}{|c|}{ Threshold } & Transition & Interior \\
\hline Section & First & Second & & \\
\hline Length & $23 \mathrm{~m}$ & $22.5 \mathrm{~m}$ & $500 \mathrm{~m}$ & $3515 \mathrm{~m}$ \\
\hline Average luminance & $288 \mathrm{~cd} / \mathrm{m}^{2}$ & $172.80 \mathrm{~cd} / \mathrm{m}^{2}$ & $114.45 \mathrm{~cd} / \mathrm{m}^{2}$ & $7.04 \mathrm{~cd} / \mathrm{m}^{2}$ \\
\hline Luminous flux intensity coefficient & 0.49 & 0.46 & 0.20 & 0.01 \\
\hline Energy consumption & $6.86 \%$ & $6.40 \%$ & $60.57 \%$ & $26.17 \%$ \\
\hline
\end{tabular}

Table 5. Calculation Results for Trabzon Tunnel

\begin{tabular}{|c|c|c|c|c|}
\hline Tunnel Zone Detail & \multicolumn{2}{|c|}{ Threshold } & Transition & Interior \\
\hline Section & First & Second & & \\
\hline Length & $20 \mathrm{~m}$ & $20 \mathrm{~m}$ & $400 \mathrm{~m}$ & $1262 \mathrm{~m}$ \\
\hline Average luminance & $300 \mathrm{~cd} / \mathrm{m}^{2}$ & $180 \mathrm{~cd} / \mathrm{m}^{2}$ & $119.84 \mathrm{~cd} / \mathrm{m}^{2}$ & $7.33 \mathrm{~cd} / \mathrm{m}^{2}$ \\
\hline Luminous flux intensity coefficient & 0.27 & 0.25 & 0.11 & 0.01 \\
\hline Energy consumption & $8.72 \%$ & $8.14 \%$ & $69.69 \%$ & $13.45 \%$ \\
\hline
\end{tabular}

$$
L_{t r}=L_{t h}(1.9+t)^{1.4} .
$$

The value of the average road surface luminance in the interior region is called the inner zone luminance $\left(L_{i n}\right)$

$$
L_{i n}=\frac{L_{e x}}{\beta} .
$$

Eq. (1)-(3) stands for the luminance of various tunnel zones. $L_{t h}, L_{20}, L_{t r}, L_{i n}$, and $L_{e x}$ are the luminance of threshold, access, transition, interior, and exit zones $\left(\mathrm{cd} / \mathrm{m}^{2}\right)$, while the $k, \beta$, and $\lambda$ are luminance conversion coefficients.

\subsection{Methodology}

The tunnel luminous flux and luminance were calculated from the information provided by the designer. The designer needs to give the detailed parameters of the tunnel. Access zone luminous flux, the tunnel's length, the speed limit of the tunnel, and the luminous flux of the luminaire used in the tunnel.
Table 2 shows the information provided by the user in this study. The length of the tunnel in meter was $7500 \mathrm{~m}$, speed limit according to the CIE88:2004 standard [8] was $80 \mathrm{~km} / \mathrm{h}$, the luminaires luminous flux used in the tunnel was $28000 \mathrm{~lm}$, the access zone luminance $L_{20}$ was $4000 \mathrm{~cd} / \mathrm{m}^{2}$, and the luminaire surface area was $0.08 \mathrm{~m}^{2}$. The proposed algorithm was implemented using the computer-aided software (Codeblock) in the $C$ programming language to calculate the luminance and luminous flux of the different tunnel zones.

Calculation of energy consumed in the tunnel zones is essential for reducing power consumption, thereby limiting the payment of excessive bills for wastage energy. In this regard, tunnel illumination design always includes energy consumption calculation. The calculation includes the selection of energy-efficient luminaires primarily, and then using some optimization computing algorithm, power consumption is calculated. In this paper, a simple algorithm is used, as illustrated in appendix A.5. 


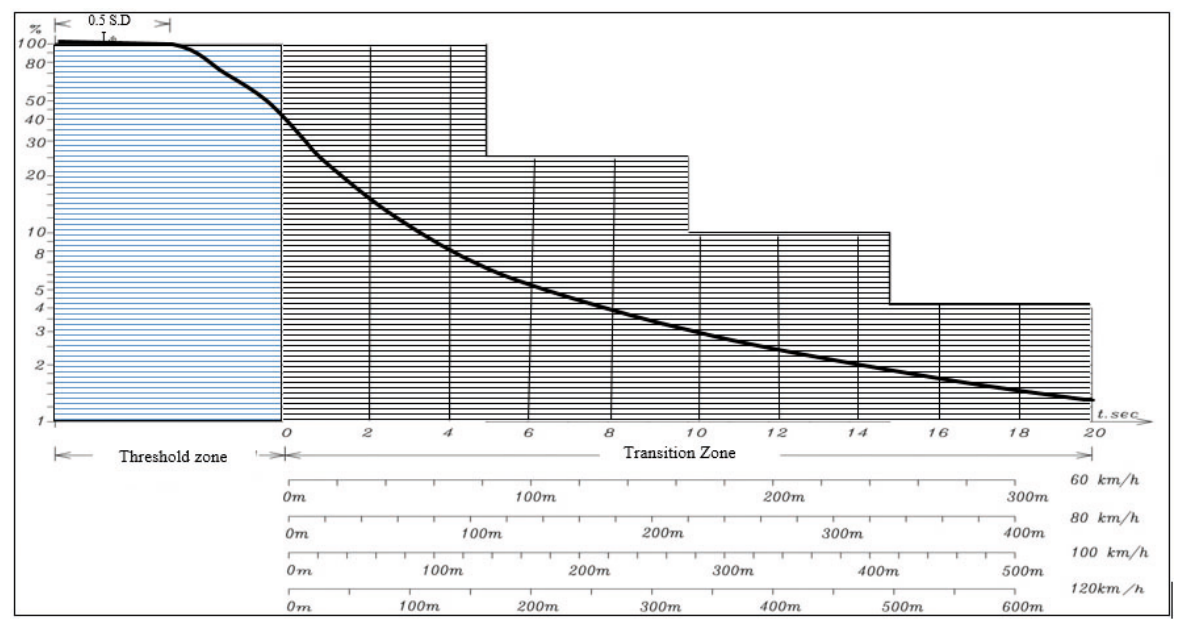

Fig. 2. The distribution of luminance in a tunnel $[8]$

The LED luminaires selected were energy efficient and good quality luminaires. LED light source was preferred due to its advantages over traditional lamps. It has a longer service lifespan of around $50000 \mathrm{~h}$, suitable correlated colour temperature (CCT), and colour rendering index (CRI).

The code in appendix A. 1 is a user-defined code that asked the tunnel lighting designer to provide the tunnel's details: the length, the access zone luminance, the speed limit, luminous flux, and surface area parameters of the luminaire in the tunnel according to the CIE88:2004 standard [8]. Once these details are provided, the stopping or the braking distance of the tunnel is calculated. Similarly, the code in appendix A. 2 has been used to find the threshold zone length in the tunnel illumination based on the calculated stopping distance and the speed limit of the tunnel. In Fig. 1, half of the threshold area was computed as $80 \%$ of the access zone luminance and is defined as the luminance value of the threshold zone. The other half part luminance value is estimated as $56 \%$ of the access zone luminance $L_{20}$.

Moreover, the transition zone luminous flux and luminance were equally determined, as shown in appendix A.3 while considering the CIE88:2004 [8] tunnel illumination standard guidelines and various speed limits. Further, appendix A.4 code shows the computation of the interior zone luminance and luminous flux using (1). The $t$ value in (1) depends on both the length and speed of the transition zone. Therefore, when all the information was given, the proposed algorithm could compute the luminous flux and luminance of the zones. Finally, appendix A.5 illustrates the algorithm for power consumption for each zone and the total power consumed in the entire tunnel.
All the above-computed information could be used by the designer for designing the tunnel [10].

\section{RESULTS}

This study aims to calculate the luminous flux and luminance of the tunnel using the detailed information provided by the user. The computation was done using an enhanced algorithm in the $C$ programming language to solve the objective function. These calculated data were used in the design optimization of the tunnel. The algorithm is presented in appendix A.

In Table 3, we can observe that the results found are similar to the CIE88:2004 [8] tunnel illumination standard values. The threshold zone length, average luminance in the first half $L_{a v}$, and the last half of the threshold zone are $40 \mathrm{~m}, 240 \mathrm{~cd} / \mathrm{m}^{2}$, and $144 \mathrm{~cd} / \mathrm{m}^{2}$, respectively. However, the average luminance of the transition and interior zones is $95.87 \mathrm{~cd} / \mathrm{m}^{2}$ and $5.80 \mathrm{~cd} / \mathrm{m}^{2}$. In contrast, the luminous flux coefficient of the threshold first, second, transition, and the interior zones are $0.27,0.25$, 0.11 , and 0.01 , respectively. The findings confirmed the efficiency of the proposed algorithm for calculating the luminance of the zones inside the tunnel.

As shown above, Table 3 compares the calculated values by the proposed algorithm and the CIE88:2004 standard values. We can see that these values are the same. Thus, the proposed algorithm can be used on different tunnel lengths to compute the luminous flux and the luminance with great accuracy.

Tables 4 and 5 show the calculation results for two study cases of Alanya [12] and Trabzon tunnels in Turkey. The results were compared with the ac- 
tual values to confirm the validity and efficiency of the proposed algorithm.

\section{CONCLUSION AND DISCUSSION}

Tunnel illumination is the critical part of tunnel design that needs keen attention while designing a tunnel. A good illumination of the tunnel helps a driver enter and leave the tunnel safely without meeting any difficulties. Therefore, a good illumination of the tunnel is necessary for this effect. In this work, tunnel illumination was designed according to the international tunnel illumination standards. Here, the goal was to develop an algorithm that will be used to compute the luminance of the tunnel zones once the designer supplies the necessary information about the tunnel. Therefore, the calculated luminance of the case study using $L_{20}$ equal $4000 \mathrm{~cd} / \mathrm{m}^{2}$. The threshold zone first, second section luminance, transition zone, and interior zone of the tunnel were obtained as $240 \mathrm{~cd} / \mathrm{m}^{2}, 144 \mathrm{~cd} / \mathrm{m}^{2}$, $95.87 \mathrm{~cd} / \mathrm{m}^{2}$, and $5.86 \mathrm{~cd} / \mathrm{m}^{2}$, respectively.

The energy consumption for the different sections was equally calculated (\%). From the results obtained for the 3 cases, it is noticed that the transition and threshold zones consume more energy. As seen in Table 5, the energy consumption for the Trabzon tunnel is $69.9 \%$, while the total energy consumption of the threshold zone is $16.86 \%$.

Despite the high level of luminance in the threshold zone, the energy consumption in this zone is less due to it small distance compared to the transition zone.

The developed algorithm in the $C$ programming language conformed to the CIE88:2004 tunnel illumination standard. Therefore, depending on different geographical conditions and the desire luminaires, the luminance value and luminous flux requirement for the threshold, transition, interior zones can be computed accurately by following the CIE88:2004 tunnel illumination standard [8]. This study aims to calculate the preliminary data for the tunnel illumination design by strictly respecting the standards guidelines and the environmental factors while considering luminaire parameters details to ease the tunnel design. Verifying the algorithm's performance was done by taking three case studies on two tunnels in Turkey and using CIE standard values. According to the numerical result obtained, it was seen that the numerical results are compatible with the graphics specified in the standard and equally the two tunnel case studies. Thus, the proposed algorithm is valid.

\section{APPENDIX A}

\section{A.1 SECTION}

\#include $<$ iostream $>$

\#include $<$ stdio.h $>$

\#define N8

\# include $<$ math.h $>$

int main(int arge, char** argv) \{

float length, length1, esk, power, Total1, Total2,

Total3, Total4, Total;

float L20;

float speedlimit, lumen, uz, area, time;

printf("enter the lenght of the tunnel(m) $\backslash n$ ");

scanf("\%f",\&length1);

printf("enter the luminance of the access zone $\left.\left(\mathrm{cd} / \mathrm{m}^{\wedge} 2\right) \backslash \mathrm{n} "\right)$;

scanf(“\%f”,\&L20);

printf("enter the speed limit for the tunnel

$(\mathrm{km} / \mathrm{s}) \backslash \mathrm{n}$ ");

scanf(“\%f”,\&speedlimit);

printf("enter the luminous flux of the luminaire to be used (lumen) \n");

scanf(“\%f",\&lumen);

printf("enter the luminaire surface area $\left.\left(\mathrm{m}^{\wedge} 2\right) \backslash \mathrm{n} "\right)$;

scanf(“\%f”,\&area);

printf("enter the luminaire power (W) $\backslash$ ");

scanf(“\%f”,\&power);

length=length 1 ;

float ars;

float distance, brake;

brake $=$ speedlimit $/ 2$;

\section{A.2 SECTION}

*/Threshold zone luminance and Power consumption/*

printf("threshold zone details $\backslash \mathrm{n}$ ");

printf("threshold zone length\%.f in metre $\backslash n$ ", brake);

printf("first\%.f metre luminance value $\% .2 \mathrm{f}(\mathrm{cd} /$ $\mathrm{m}^{\wedge} 2$ ).. $\mathrm{n}$ ", brake/2, L20*0.06);

ars $=(3.14 * \mathrm{~L} 20 * 0.06 *$ area $) /$ lumen;

Total1 $=$ ars $*$ brake $/ 2$;

printf("first half luminous flux of the threshold zone $\% .2 \mathrm{f}$. In", ars);

printf("the last threshold zone $\% .2 \mathrm{f}$ metre luminance value $\% .2 \mathrm{f}\left(\mathrm{cd} / \mathrm{m}^{\wedge} 2\right)$. $\backslash \mathrm{n}$ ”, brake $/ 2$, L20*0.036); 
ars $=(3.14 * \mathrm{~L} 20 * 0.056 *$ area $) /$ lumen; printf("threshold zone luminous flux\%.2f. $\backslash n$ ", ars);

Total2 $=($ brake/2)*ars; length=length-brake;

\section{A.3 SECTION}

*/Transition zone luminance and Power consumption/*

printf("transition zone details $\backslash n$ ");

if(speedlimit $<=60$ )

\{

float esk, time, Ltr, test;

esk $=0.3$;

time $=$ esk/speedlimit;

time $=$ time $* 60$;

$\mathrm{Ltr}=(\mathrm{L} 20 * 0.06) * \operatorname{pow}((1.9+$ time $),-1.4)$;

$\mathrm{Ltr}=(\operatorname{Ltr}+(\mathrm{L} 20 * 0.036)) / 2$;

printf("transition zone luminance $\% .2 \mathrm{f} \mathrm{cd} /$ $\mathrm{m}^{\wedge} 2 . \ln$ ”, Ltr);

ars $=(3.14 *$ Ltr*area $) /$ lumen;

length=length-(esk*1000);

printf("transition zone luminous flux\%.2f. $\backslash n$ ", ars);

printf(“transition zone length\%.2F", esk*1000);

Total3 $=\left(\right.$ esk $\left.^{*} 1000\right) *$ ars; $\}$

if(speedlimit $>60 \& \&$ speedlimit $<=80$ )

\{

float esk, time, Ltr, test;

esk $=0.4$

time $=$ esk/speedlimit;

time $=$ time $* 60$;

$\mathrm{Ltr}=(\mathrm{L} 20 * 0.06) * \operatorname{pow}((1.9+$ time $),-1.4)$;

$\mathrm{Ltr}=(\mathrm{Ltr}+(\mathrm{L} 20 * 0.06)) / 2$;

printf("transition zone luminance $\% .2 \mathrm{f}$

$\mathrm{cd} / \mathrm{m}^{\wedge} 2$. . nn", Ltr);

ars $=(3.14 *$ Ltr*area $) /$ lumen;

length=length-(esk*1000);

printf("transition zone luminous flux\%.2f. $\backslash n$ ", ars);

printf(“transition zone length\%.2F", esk*1000);

Total3 $=($ esk*1000)*ars; $\}$

if(speedlimit $>80 \& \&$ speedlimit $<=100$ )

\{

float esk, time, Ltr, test;

esk $=0.5$;

time $=$ esk/speedlimit;

time $=$ time $* 60$;

$\mathrm{Ltr}=(\mathrm{L} 20 * 0.06) * \operatorname{pow}((1.9+$ time $),-1.4)$;

$\mathrm{Ltr}=(\operatorname{Ltr}+(\mathrm{L} 20 * 0.06)) / 2$; printf("transition zone luminance $\% .2 \mathrm{f}$ $\mathrm{cd} / \mathrm{m}^{\wedge} 2 . \mathrm{n}$ ", Ltr);

ars $=(3.14 *$ Ltr*area $) /$ lumen;

length=length-(esk*1000);

printf("transition zone luminous flux\%.2f. $\backslash n$ ", ars);

printf(“transition zone length\%.2f", esk*1000);

Total3 $=($ esk*1000)*ars; $\}$

if(speedlimit $>100 \& \&$ speedlimit $<=120$ )

\{

float esk, time, Ltr, test;

esk $=0.6$;

time $=$ esk/speedlimit;

time $=$ time $* 60$;

$\mathrm{Ltr}=(\mathrm{L} 20 * 0.06) * \operatorname{pow}((1.9+$ time $),-1.4)$;

$\mathrm{Ltr}=(\operatorname{Ltr}+(\mathrm{L} 20 * 0.06)) / 2$;

printf("transition zone luminance\%.2f cd/ $\mathrm{m}^{\wedge} 2$. . $n$ ", Ltr);

ars $=(3.14 *$ Ltr*area $) /$ lumen;

length=length-(esk*1000);

printf("transition zone luminous flux\%.2f. $\backslash n$ ", ars);

printf(“transition zone length\%.2F", esk*1000); Total $3=($ esk $* 1000) * \operatorname{ars} ;\}$

\section{A.4 SECTION}

*/Interior zone luminance and Power consumption/*

float Lin, Lin_length, Totalpower;

printf("interior zone details $\backslash n$ ");

$\mathrm{Lin}=(\mathrm{L} 20 * 0.06) *$ pow $((1.9+$ time $),-1.4)$;

printf(“interior zone luminance $\% .2 \mathrm{f} \mathrm{cd} / \mathrm{m}^{\wedge} 2 \backslash \mathrm{n}$ ”, Lin);

ars $=(3.14 *$ Lin*area $) /$ lumen;

printf("interior zone luminous flux \%.2f. \n ", ars);

Lin_length $=(($ length $))$;

printf("interior zone length\%2.fln", Lin_length);

Total4=ars*Lin_length;

\section{A.5 SECTION}

*/Total Power consumption in the Tunnel/*

Total=Total1+Total2+Total3+Total4;

Totalpower=Total ${ }^{*}$ power;

printf("Threshold zone first section installed power\%.2f(W) $\backslash$ n", Total1*power);

printf("Threshold zone second section installed power\%.2f(W) $\backslash n$ ", Total2*power); printf("Transition zone installed power\%.2f (W) $\backslash$ n “, Total3*power); 
printf("Interior zone last section installed $\% .2 \mathrm{f}$ (W) $\backslash n$ ", Total4*power);

*/ expressing the Total Power consumption in the Tunnel in Percentage/*

printf("Percentage installed power of the Tunnel is the $\% .2 \mathrm{f}$ of the Threshold zone first section installed power \n",(((Total1*power $) /$ Totalpower)*100));

printf("Percentage installed power of the Tunnel is $\% .2 \mathrm{f}$ of the Threshold zone second section installed power \n",(((Total2* power $) /$ Totalpower)*100));

printf("Percentage installed power of the Tunnel is $\% .2 \mathrm{f}$ of the Transition zone installed power .In",(((Total3*power)/Totalpower)*100));

printf("Percentage installed power of the Tunnel is $\% .2 \mathrm{f}$ of the Interior zone last section installed. $\backslash n$ ", (( ( Total4* power $) /$ Totalpower)*100)); return $0 ;\}$

\section{REFERENCE}

1. T. 4-35. Commission International de l'Eclairage, Guide for the Lighting of Road Tunnels and Underpasses // CIE PubLination 88-2004, 2004, Vol. 88, pp. 1-34.

2. Li S. G., Tu G., and Zhou Q. An optimal design model for tunnel lighting systems // Optik (Stuttg)., 2021, Vol. 226, p. 165660.

3. Pachamanov A. and Pachamanova D., Optimization of the light distribution of luminaries for tunnel and street lighting // Eng. Optim., 2008, Vol. 40, \#1, pp. 47-65.

4. Yang C., Xiao Y., Xu Y.H., and Fan S.J. Parameters optimization and simulation of highway tunnel symmetric-luminaire-distribution lighting with LED, 2017, pp. 1153-1164.

5. Parise G., Martirano L., Parise L., Carrarini L., and Mitolo M. The Electrical Systems of Roadway Tunnels: Safety Design and Ecomanagement // IEEE Trans. Ind. Appl., 2015, Vol. 51, \#2, pp. 1920-1927.

6. Leitao Ś., Pires E.J. S., and De Moura Oliveira P.B. Road Tunnels Lighting using Genetic Algorithms // IEEE Conference. https://ieeexplore.ieee.org/ document/5352835 (accessed Jun. 18, 2021).

7. Cengiz M. S., Simulation and Design Study For Interior Zone Luminance In Tunnel Lighting // Light \& Engineering, 2019, Vol, 27, \#2, pp. 42-51.

8. International Commission on Illumination, Guide for the lighting of road tunnels and underpasses, p. 40, 2004.
9. Chen M. J., Tseng W.H., Liao H.Y., Chen S.Y., Ma H.Y., and Lee H.Y. Design of Counter Beam Lights for Road Tunnels Lighting with CIE Regulations // 2019 IEEE Eurasia Conf. IOT, Commun. Eng. ECICE2019, pp. 550-551, 2019.

10. Abdul Salam A.O. Automation and control of DIA transportation tunnel // 2007 Mediterr. Conf. Control Autom. MED, no. July 2007, 2007.

11. B. Li and Yang T., "The Design of Tunnel Lighting Intelligent Control System," Proc. - 3rd Int. Conf. Intell. Transp. Big Data Smart City, ICITBS2018, vol. 2018-Janua, pp. 592-595, 2018.

12. Aykut E. M. et al., Tunnel Lighting," Yildiz Technical University, Istanbul, 2006.

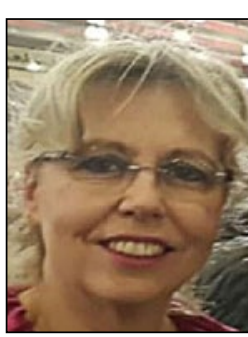

\section{Canan Perdahci,}

Ph.D. In 1992, she joined the Department of Electrical Engineering, University of Kocaeli, as a Lecturer, and in 2019 became an Associate Professor. Her current research interests include LED illumination, UVC, power quality, tunnel illumination, road illumination

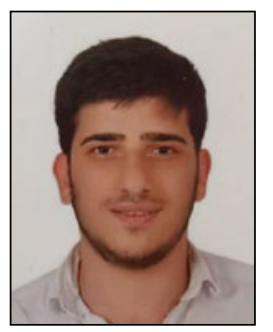

\section{Onur Hasanoglu,}

B. Sc. He is currently a master student in the Department of Electrical Engineering, University of Kocaeli and student in the Department of Computer Engineering, University of Kocaeli. His current research interests include LED illumination, UVC, tunnel illumination, road illumination, $C$ programming, algorithm development, and smart cities

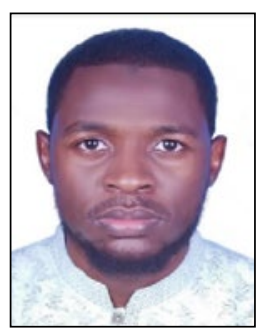

\section{Yahya Sogodok,}

B. Sc. He is currently a master student in the Department of Electrical Engineering, University of Kocaeli. His current research interests include LED illumination, UVC, tunnel lighting, road lighting 